\title{
Mindfulness-based cognitive therapy for depression: trends and developments
}

\author{
Meagan B MacKenzie' \\ Nancy L Kocovski \\ 'Department of Psychology, Ryerson \\ University, Toronto, ON, Canada; \\ ${ }^{2}$ Department of Psychology, Wilfrid \\ Laurier University, Waterloo, \\ ON, Canada
}

This article was published in the following Dove Press journal:

Psychology Research and Behavior Management

19 May 2016

Number of times this article has been viewed

\begin{abstract}
Mindfulness-based cognitive therapy (MBCT) was developed as a psychological intervention for individuals at risk of depressive relapse. Possible mechanisms of change for this intervention are in line with its theoretical underpinnings, and include increases in mindfulness and/or decreases in negative repetitive thoughts. This review provides an overview of current trends in MBCT research, including efficacy and questions regarding the specific effects of MBCT in light of recent comparisons with structurally equivalent control conditions, mechanisms of change, and moderators of treatment outcome. In addition, future directions are discussed, such as challenges with training an adequate number of therapists and disseminating this therapy.
\end{abstract}

Keywords: MBCT, efficacy, mechanisms of change, dissemination, moderators

\section{Introduction}

Major depressive disorder (MDD) is characterized by pervasive and persistent low mood and lack of interest in normally pleasurable activities. These features are accompanied by a range of additional symptoms, such as poor appetite or overeating, insomnia or fatigue, low energy, low self-esteem, feelings of worthlessness or guilt, difficulty concentrating, and recurrent thoughts of death. ${ }^{1}$ MDD is highly prevalent, and is associated with considerable impairment in social, occupational, and other important areas of functioning, leading to significant economic and service burdens worldwide. ${ }^{2}$ Depression has become a significant health issue, partly because it tends to run a relapsing course. ${ }^{3}$ At least half the individuals who have experienced one major depressive episode are at risk of one or more additional episodes, and $80 \%$ of people with a history of two episodes are likely to have another recurrence. ${ }^{1,4}$ As a result of the challenging nature of treating this disorder, mindfulness-based cognitive therapy (MBCT) was developed to reduce depressive relapse.

\section{Mindfulness-based cognitive therapy}

MBCT is a manualized group-therapy program that was developed by Zindel Segal, John Teasdale, and Mark Williams, and was designed to address vulnerabilities to depressive relapse for individuals with recurrent MDD. ${ }^{5,6} \mathrm{MBCT}$ draws from cognitive behavior therapy (CBT) and traditional mindfulness practices, as articulated by KabatZinn within his mindfulness-based stress-reduction (MBSR) program. ${ }^{7}$ Mindfulness is defined as nonevaluative present-moment awareness, and is used within MBCT to help
Correspondence: Nancy Kocovski Department of Psychology, Wilfrid Laurier University, 75 University Avenue West, Waterloo, ON N2L 3C5, Canada $\mathrm{Tel}+\mathrm{I} 5198840710$ ext 3519

$\mathrm{Fax}+\mathrm{I} 5197467605$

Email nkocovski@wlu.ca 
individuals disengage from their ruminative thoughts and to promote detachment and decentering from depression-related thoughts and feelings. ${ }^{8}$

MBCT was developed from a model of cognitive vulnerability that stated that individuals who experience depressive relapses have different patterns of negative thinking from those who do not experience relapses. According to the model, for those who have previously experienced relapses, small negative mood shifts may produce recurrence, because they can activate patterns of depressive thinking that are similar to the thoughts experienced in previous depressive episodes. ${ }^{5}$

MBCT encourages individuals with MDD to become more aware of their internal events (ie, thoughts, feelings, and bodily sensations) and to change the ways in which they relate to these thoughts. For example, individuals are encouraged to view their thoughts as passing events in the mind, rather than treat them as reality. Disengaging from automatic negative cognitive patterns, such as rumination, reduces the future risk of relapse.

\section{The present review}

Given the popularity of third-wave behavior therapies characterized by such concepts as mindfulness and acceptance, a review of current trends and future directions of MBCT is required to provide an overview of the state of the research to date. This review presents current research pertaining to the efficacy of MBCT, especially as it relates to comparisons with active-control conditions (ACCs), mechanisms of change for this therapy, and moderators of treatment outcome, and concludes with a discussion of dissemination. This narrative review of trends and developments in the field aims to inform clinical practice and provide directions for future research.

\section{Efficacy of MBCT}

There is consistent empirical evidence in support of using MBCT to decrease the risk of depressive relapse. Beginning with the initial trial of this intervention in 2000, 145 individuals who were in remission or recovery from major depression were randomized to either an 8-week course of MBCT or treatment as usual (TAU). Participants were followed for 1 year, and the results indicated that of those randomized to MBCT, only $40 \%$ relapsed during the follow-up period compared to $66 \%$ of those in the TAU group. ${ }^{9}$ In 2008 , a follow-up study of 123 patients in remission or recovery from major depression who were randomized to either maintenance antidepressant medication or medication plus MBCT showed that the MBCT group had a significantly lower relapse rate over 15 months than the medication-alone group (47\% vs $60 \%$ ). Also within this study, MBCT was associated with a reduction in depressive and comorbid symptoms and an increase in quality of life. ${ }^{10}$ These results were replicated more recently in 2010 by Segal et al. ${ }^{11}$

In addition to empirical trials, MBCT has emerged as an effective therapy option in systematic reviews and metaanalyses. A systematic review conducted by Coelho et al ${ }^{12}$ in 2007 evaluated the results of four studies that compared MBCT plus TAU to TAU alone..$^{9,13-16}$ The results of this review culminated in the conclusion that for individuals who have experienced three or more depressive episodes, MBCT is a positive adjunct to TAU. The authors did qualify this conclusion by noting that there may be issues with control groups, so this conclusion should be interpreted with caution. Specifically, the trials in this review compared MBCT to TAU, which can only indicate whether some kind of additional treatment action results in a positive change. The trials did not compare MBCT to other nonpharmaceutical treatments or similar group settings, making it difficult to identify any specific effects of MBCT.

Specifically, in their 2015 systematic review and metaanalysis, Clarke et al examined the effects of nonpharmacological interventions on depressive relapse prevention. ${ }^{17}$ Their review culminated in the conclusions that at 12 months, MBCT, CBT, and interpersonal psychotherapy were each associated with a significant reduction in relapse compared to active and inactive controls. However, the authors concluded that MBCT's efficacy may be 1) limited to participants who had recovered from their depression via pharmacological means and 2) a nonspecific effect of treatment. Considering the homogeneity of the trials in this review (ie, the structure, content, and delivery of MBCT) and that the majority of participants were individuals who responded well to pharmacological treatment for depression, the literature could be further developed by testing MBCT with diverse patient populations and varying the treatment delivery.

One of the newer trends in the study of MBCT is the extension of examining depressive relapse as the primary outcome variable to examining depressive symptoms as an outcome variable. In 2014, Strauss et al ${ }^{18}$ conducted a meta-analysis of randomized controlled trials evaluating mindfulness-based interventions for individuals with current anxiety or major depression. This analysis of 12 studies demonstrated a significant effect associated with MBCT compared to control conditions and MBSR for primary symptom severity for those with current depression. This particular analysis provides strong evidence for the effectiveness of 
MBCT with individuals who are currently experiencing a major depressive episode, in addition to the strong evidence in support of MBCT for those who are currently in remission.

\section{Efficacy of MBCT compared to active-control groups}

Since Coelho et $\mathrm{al}^{12}$ conducted their systematic review in 2007, new research comparing MBCT to more appropriate control groups has been published. There are early indications that although MBCT is effective, it may not be superior to control groups intended to be structurally equivalent. Six studies that have compared MBCT to carefully designed ACCs have demonstrated mixed findings for significant group differences for depressive relapse ${ }^{19-21}$ and depressive symptoms. $^{22-24}$

Studies that included depressive relapse as a primary outcome variable have typically indicated no difference between MBCT and ACCs. For instance, in a large-sample randomized controlled trial with participants who had had three or more episodes of recurrent MDD, Williams et al compared MBCT to cognitive psychological education and TAU. ${ }^{21}$ The results of this trial showed that MBCT was superior in preventing relapse for individuals who had experienced childhood trauma, but that MBCT did not show a significant advantage over the active-control or TAU groups for the whole sample. The authors suggested that the significance of childhood trauma was in line with previous studies showing that MBCT had more positive effects for vulnerable populations. . $^{9,11,14}$

Moreover, in 2015 Shallcross et al compared MBCT to a structurally equivalent ACC for patients in remission from MDD with residual depressive symptoms. ${ }^{20}$ A total of 92 participants were randomly assigned to either condition, and the results showed that MBCT did not differ from the ACC on primary or secondary outcome variables, including rate of depression relapse, symptom reduction, and life satisfaction.

Conversely, one study has shown more positive results comparing MBCT to an ACC. Meadows et al examined a sample of approximately 200 nondepressed individuals with a history of three or more depressive episodes to determine if MBCT was superior to an ACC in reducing depressive relapse. ${ }^{19}$ Results showed that MBCT combined with depression-relapse active monitoring was superior to depression-relapse active monitoring alone in reducing depressive relapse/recurrence over 2 years of follow-up.

With respect to the trend of measuring depressive symptoms as the primary outcome variable, MBCT also has mixed outcomes relative to ACCs. A recent randomized controlled trial published in 2016 by Eisendrath et al compared MBCT to a health-enhancement program (physical fitness, music therapy, and nutrition education) in addition to TAU in a sample of individuals with treatment-resistant depression. ${ }^{24}$ Results showed that relative to the health-enhancement program, MBCT was associated with a significant reduction in depression-symptom severity and a significant increase in treatmentresponse rates. In 2015, Chiesa et al compared MBCT to a psychoeducation control group with positive results. ${ }^{23} \mathrm{~A}$ total of 43 patients with MDD who had not achieved remission following the use of antidepressants were randomly assigned to receive either $\mathrm{MBCT}$ or a psychoeducation program designed to be structurally equivalent. Patients in the MBCT group had significantly larger improvements in depressive symptoms, as well as for quality of life and mindfulness.

Conversely, Manicavasgar et al randomly assigned a total of 45 participants who met criteria for current MDD to either an 8-week MBCT or CBT group. ${ }^{22}$ Both groups reported significant improvements in depression and anxiety; however, there were no group differences in outcomes for the two treatment conditions. Perhaps these discrepant findings were related to the patient population, ie, those who had not responded previously to pharmacological treatments. Many of the previous trials included individuals who had used antidepressants to assist them in their remission. This variation in findings highlights the need for additional study. In line with this need, one additional study protocol has been identified that compares MBCT to a psychoeducational intervention. ${ }^{25}$ In summary, additional research comparing MBCT to ACCs must be conducted, in order to evaluate further the efficacy of this treatment.

\section{Evaluating mechanisms of change}

A current trend in psychotherapy research in general is the identification of mechanisms of change for new and existing interventions. ${ }^{26,27}$ Despite the mixed findings when comparing MBCT to ACCs, the preliminary evidence of MBCT's effectiveness warrants this type of research, and few studies have directly examined how and why MBCT works for recurrent depressive episodes. The examination of the mechanisms by which this intervention is effective can aid in understanding the processes that lead to therapeutic change, and can aid in further development and refinement of MBCT. Additionally, this understanding can help in identifying patients for whom this therapy would benefit and perhaps to shed light on the disparate findings mentioned earlier.

Two recent systematic reviews have been conducted to examine mechanisms of change underlying MBCT. In a 2015 review, van der Velden et al evaluated the state of the research 
in this area and reviewed 23 clinical trials of MBCT for the treatment of recurrent MDD. ${ }^{28}$ Studies were included if they were clinical trials examining meditation or mechanisms in MBCT for MDD, the participants were 18 years or older, and MBCT was conducted in accordance with the manual. ${ }^{29}$ The authors examined several theoretical mechanisms of change relevant to depression that were based on the MBCT manual: increased mindfulness, meta-awareness (ie, the mind's ability to observe itself), self-compassion, decreased rumination, worry, and cognitive reactivity. Of the 23 studies included, 12 trials showed that MBCT-treatment outcome was associated with or predicted by changes in mindfulness, rumination, worry, self-compassion, decentering, and/or meta-awareness. Two studies found that increased mindfulness mediated treatment outcome, two studies found decreased rumination mediated outcome, and two studies found decreased worry mediated outcome. Lastly, one study showed that increased self-compassion mediated relapse risk and reduced the relationship between cognitive reactivity and relapse risk. There were two trials that failed to find any effect for mindfulness or rumination, and the authors suggested that this may have been due to a lack of therapist competence or fidelity to the MBCT manual. Few trials reported on therapist adherence; therefore, it is difficult to know whether a lack of treatment fidelity could be a contributing factor to a lack of effect for mindfulness and/or rumination. This review culminated in the conclusion that changes in cognitive variables, such as mindfulness, worry, meta-awareness, and self-compassion, are associated with reductions in relapse risk and depressive symptoms, and thus may be mechanisms of action for the positive effects of MBCT.

A systematic review and meta-analysis conducted by $\mathrm{Gu}$ et al in $2015^{30}$ evaluated mechanisms of action underlying both MBCT and MBSR. Inclusion criteria were different from van der Velden et al's review, ${ }^{28}$ ie, that studies were included if their outcome variables assessed mental health and well-being, rather than specifically for MDD. In addition, studies were included even if they used adapted versions of MBCT or MBSR. For inclusion, studies must also have included MBCT or MBSR in a mediation analysis. These two mindfulness-based approaches were collapsed into one intervention category. A total of 20 studies were included in this review, and of these only nine included depressive symptoms as an outcome variable. Other outcomes included anxiety, stress, mood state, quality of life, and anger expression. The 20 studies were all included in the narrative review examining mechanisms of change, and then were further broken down using two-stage meta-analytic structural equation modeling to determine whether particular mechanisms of change mediated the impact of MBCT and/or MBSR on clinical outcomes. Twelve studies were included in the quantitative analysis of mindfulness, and six studies were included in the quantitative analysis of repetitive negative thinking. The results of the narrative review showed strong and consistent evidence for cognitive and emotional reactivity, moderate and consistent evidence for mindfulness and repetitive negative thought, and preliminary but insufficient evidence for selfcompassion and psychological flexibility as mechanisms of change within mindfulness-based interventions for clinical and nonclinical outcomes. The results of the two modeling analyses showed that both mindfulness and repetitive negative thought were significant mediators of the effect of MBCT/ MBSR on mental health outcomes, including anxiety, depressive symptoms, general psychopathology, stress, and negative affect. These findings provide evidence that mindfulness is likely an influential factor in the effectiveness of MBCT for psychopathology.

The identification of mechanisms of change and mediators in psychotherapy research is valuable for providing an evidence-based explanation of how treatments work. Understanding the processes by which MBCT works will assist in potentially refining the intervention. The existing research examining mechanisms of change in MBCT have been summarized in two recent systematic reviews. Both of these papers provided strong evidence that increased mindfulness and decreased negative repetitive thought (ie, rumination) are processes that mediate the association among mindfulnessbased interventions and treatment outcome. There is also support for such mechanisms as meta-awareness, selfcompassion, and cognitive reactivity; however, the number of studies examining these variables is more limited. It is encouraging to see that the mechanisms that emerge as significant mediators are theoretically in line with the development of MBCT; however, an important line of future research will be to continue to design rigorous studies that include assessments over the course of treatment, in order to obtain consistent high-quality evidence for potential mediators. ${ }^{26,27}$ In 2015, van der Velden and Roepstorff discussed the lack of literature examining the underlying neural mechanisms of MBCT. ${ }^{31}$ They appealed to clinicians and neuroscientists to collaborate, in order to understand further the neural processes by which MBCT works. These types of investigations will certainly assist in a greater understanding of MBCT and in predicting clinical outcomes. 


\section{Moderators of treatment outcome}

Along with the examination of mechanism of change, there have been a few studies that investigated possible moderators of MBCT outcome. Early research in 2000 and 2004 by Teasdale et al found that for patients with three or more previous episodes of major depression, MBCT significantly decreased the risk of relapse., ${ }^{9,14}$ This effect was not found for participants who had had fewer depressive episodes. Kuyken et al in 2010 evaluated moderating variables, including demographics, severity of depression, and depression recurrence, and found that sex was a significant moderator, such that female participants fared better on a depression inventory at 15-month follow-up compared to males. ${ }^{32}$ The authors did note that there was a very small number of males in the study, so this result should be interpreted with caution, especially given that no significant sex effects had been found in earlier evaluations. ${ }^{9,14}$ None of the other moderators significantly predicted MBCT outcome. Finally, as noted earlier, MBCT was found to be more effective than an ACC for those who had experienced childhood trauma, but not the rest of the sample; ${ }^{21}$ therefore, the presence of a history of childhood trauma or other variables causing increased vulnerability may moderate outcome, but replication is warranted before drawing firm conclusions.

\section{Dissemination}

Taken together, the evidence points to MBCT as an empirically supported intervention that reduces the risk of relapse in individuals with recurrent depression (notwithstanding the recent comparisons with structurally equivalent $\mathrm{ACCs}$ ). In order to fully optimize the use of this treatment, there must be a critical mass of trained practitioners to deliver it. There is a concern that dissemination is challenged by difficulties in implementing this program in a widespread manner and training a sufficient number of MBCT practitioners. ${ }^{33}$

Five-day intensive training courses in MBCT are available in several locations, such as Toronto's Centre for Mindfulness Studies (www.mindfulnessstudies.com) and the Omega Institute in New York State (www.eomega.org). In order to be trained in the delivery of MBCT, clinicians must be licensed clinicians in mental health, clinicians in training, or researchers interested in learning more about this topic. Participants must also be familiar with CBT, depression and related mood disorders, and running group-therapy sessions. In addition, Segal et a ${ }^{29}$ require MBCT therapists to have their own regular, daily mindfulness practice. Their justification is that patients will likely have difficulties with their own practices, and practitioners will be better able to help if they have more than just an intellectual knowledge of mindfulness. Adherence to a daily practice is critical: in a sample of patients with a history of three or more major depressive episodes completing an MBCT program, Crane et al showed that those who completed formal mindfulness practices at home 3 or more days a week were half as likely to have depressive relapse as those who engaged in less frequent formal practice. ${ }^{34}$ These results were not found for informal mindfulness practice, which typically includes present-moment awareness and focus on daily activities, such as eating or walking. Ongoing daily formal mindfulnessmeditation practice (ie, setting aside a particular time to sit and meditate) may be difficult for patients and even some clinicians to develop and maintain, and this prerequisite may make it challenging for practitioners to sign up for training and/or to continue to use MBCT in their own clinical practice.

In order to lead an MBCT program, facilitators must have a formal mindfulness practice and be completely aligned with a mindfulness approach, in order to maintain treatment integrity. Given that these are unique requirements, it is necessary to study the training that is delivered to the clinicians, in addition to conducting usual outcome studies. Gibbons et al examined and described their own training program, which they developed to address the unique needs of MBCT facilitators. ${ }^{35}$ Ten facilitators with little-to-no mindfulnessmeditation experience participated in the training that included a 2-day retreat, a 4-month period devoted to cultivating a mindfulness practice, and a 5-day MBCT-training program. Following training, the facilitator participants led trial groups with healthy volunteers, and their performance was assessed by both the volunteers and external reviewers. There was also ongoing contact between the facilitators and the training staff as the groups were conducted. Of six facilitators who were evaluated by the external reviewer, four demonstrated treatment adherence to the majority of assessed skills. The volunteer-group participants rated the facilitators as effective and found the groups engaging. In sum, MBCT training is not only a long and involved process but also requires continual contact with the trainees, in order to ensure adherence to MBCT principles and to support the development of mindfulness practices.

A positive development in dissemination is MBCT becoming the treatment of choice for recurrent depression in the UK National Health Service (NHS). The more widespread use of MBCT in the UK can be credited to the Oxford Mindfulness Centre within Oxford University's Department of Psychiatry, as well as the Mental Health Foundation. The Mental Health Foundation released the report Be Mindful 
in 2010 advocating for the use of MBCT; however, they noted that general practitioners reported that they only had limited access to this treatment, and often chose to prescribe medications, despite believing other options may be more effective. ${ }^{36}$ Because the NHS now recommends MBCT, there is a greater need to provide appropriate and ongoing training that also addresses the need to provide cheap and effective interventions. A balance must be sought between the financial necessities of such a system and providing training of high fidelity and adherence to the original program, and Marx et al developed such a training program to address this issue. ${ }^{37}$ This program consisted of initial training, interviews with training staff, supervised cofacilitation of a group, retreats, and 2-day workshops spread across the year, and results were positive. This training is ongoing in the UK, and is promising for the continued use of MBCT in the NHS.

Given that MBCT is now being integrated into national health policies, it is necessary to consider its cost-effectiveness and whether MBCT has an effect on burden of care. In the UK, Kuyken et al examined cost-effectiveness as one facet of a larger study comparing MBCT to maintenance antidepressant treatment for depressive relapse. ${ }^{38}$ The authors found no evidence to support MBCT being superior to the antidepressants, and furthermore found no evidence that MBCT was more cost-effective than medication. Despite the lack of support for MBCT being a cost-effective alternative to pharmacological interventions, it may lessen the burden of care in primary health care services. Kurdyak et al examined administrative health data from Ontario, Canada to determine whether individuals considered high utilizers of primary health care use fewer resources following exposure to MBCT. ${ }^{39}$ The authors examined health care utilization before and after individuals received either MBCT group therapy or traditional non-MBCT group therapy. The results indicated that MBCT was associated with a significant reduction in utilization for nonmental health care, in nonpsychiatrist specialist visits, and in psychiatrist visits.

Another way in which the developers of MBCT have been able to disseminate their treatment protocol was to present it via the Internet. First, there is an online training portal (www.mindfulnoggin.com) designed to support practitioners in using MBCT skills with their clients. Coupled with this service for therapists, there is also an Internet-based selfhelp version of MBCT. This version, Mindful Mood Balance (MMB), ${ }^{40}$ was evaluated using a group of 100 recurrently depressed patients, and the program was associated with significant reductions in depressive symptoms at 8 weeks. ${ }^{41}$
In a quasiexperimental study, MMB was also compared to TAU in a sample of over 200 recurrently depressed patients. ${ }^{42}$ The patients who used MMB reported a significant reduction in their depression severity, and there was evidence that these treatment gains were maintained in the 6-month follow-up. Patients also reported significant changes in rumination and mindfulness.

Furthermore, the authors have written self-help books to assist in disseminating MBCT. The first, The Mindful Way Through Depression: Freeing Yourselffrom Chronic Unhappiness ${ }^{43}$ is a self-led MBCT program for self-identified depressed individuals. Another book, Mindfulness: A Practical Guide to Finding Peace in a Frantic World, ${ }^{44}$ uses an 8-week program and instructs readers in mindfulness meditation and principles of MBCT to reduce general stress and anxiety and enhance their personal resilience. To date, there have been few evaluations of these books, and given the inherent difficulties in conducting self-help research, this is not surprising. Taylor et al examined The Mindful Way Through Depression: Freeing Yourself from Chronic Unhappiness in a sample of student participants who self-identified as experiencing stress, anxiety, or low mood. ${ }^{45}$ Compared to a waiting-list control group, those who used the book reported significantly reduced depressive symptoms, anxiety, and stress. Participants in the book group also reported significant improvements in life satisfaction, mindfulness, and self-compassion. A valuable future line of inquiry will be to continue to conduct efficacy studies of these workbooks. A similar concern to the question of efficacy of self-help methods is that there is very little evidence to support the effectiveness of MBCT when delivered in the community. One group of researchers successfully recruited individuals with partially remitted depression using community advertisements, and showed that MBCT was associated with improved emotional reactivity to social stress, and that negative repetitive thought and increases in mindfulness were significant mechanisms of change in this sample. ${ }^{46,47}$ Despite the promising findings, additional evidence is required.

\section{Future directions and conclusion}

MBCT was developed as a psychological intervention for individuals at risk of depressive relapse. As promising as the evidence in support of MBCT is, it is not without limitations. First, the efficacy literature includes some disparate findings for the efficacy of MBCT compared to structurally equivalent ACCs. There are few of these studies; therefore, additional research is warranted prior to coming to any strong conclusion. In addition, the evidence for mechanisms of change remains limited. There is growing evidence for increased 
mindfulness and decreased negative repetitive thought as mediators of change; however, rigorous treatment-dismantling studies are still required to make clear and confident conclusions about how MBCT works. Further, considering the limited research on treatment moderators, expanding the research focused on for whom MBCT works best is another worthwhile future direction.

Consumers of the research literature in this area are concerned that many of the controlled trials are being conducted by the developers of MBCT. While many articles are authored by one or more of the original MBCT developers, it does appear that the state of the empirical literature is expanding. New impartial researchers are examining more varied outcomes and more diverse mechanisms of change. As such, these researchers are becoming increasingly involved with this intervention's efficacy evaluations, and this will likely reduce existing concerns of bias. Nonetheless, additional independent evaluations are encouraged, as this can only serve to improve the existing research base.

As a result of these limitations, it is difficult to draw strong conclusions in systemic reviews and/or meta-analyses to determine the specific effects of MBCT and to determine precise effects for homogeneous groups of patients. Despite these shortcomings inherent in evaluations of new treatments, the existing evidence appears to be supportive of MBCT. A logical step in the continuation of this research is to ensure that an adequate number of clinicians and researchers are trained in MBCT, and to encourage them to conduct controlled trials with appropriate comparison conditions. Additional research on MBCT delivered in the community as well as through self-help modalities may also be helpful, especially considering the issues of dissemination. Less resource-heavy self-help treatments, such as the Internetbased MMB, will provide mental health consumers with effective therapy without heavily taxing clinicians.

\section{Disclosure}

The authors report no conflicts of interest in this work.

\section{References}

1. American Psychiatric Association. Diagnostic and Statistical Manual of Mental Disorders. 5th ed. Washington: American Psychiatric Publishing; 2013.

2. Üstün TB, Ayuso-Mateos JL, Chatterji S, Mathers C, Murray CJ. Global burden of depressive disorders in the year 2000. Br J Psychiatry. 2004;184:386-392.

3. Keller MB, Klerman GL, Lavori PW, Coryell W, Endicott J, Taylor J. Long-term outcome of episodes of major depression: clinical and public health significance. JAMA. 1984;252:788-792.
4. Kupfer DJ, Frank E, Wamhoff J. Mood disorders: update on prevention of recurrence. In: Mundt C, Goldstein MJ, Hahlweg K, Fiedler P, editors. Interpersonal Factors in the Origin and Course of Affective Disorders. London: Royal College of Psychiatrists; 1996:289-302.

5. Segal ZV, Williams JM, Teasdale JD. Mindfulness-Based Cognitive Therapy for Depression. New York: Guilford Press; 2012.

6. Williams JM, Russell I, Russell D. Mindfulness-based cognitive therapy: further issues in current evidence and future research. J Consult Clin Psychol. 2008;76:524-529.

7. Kabat-Zinn J. Mindfulness-based interventions in context: past, present, and future. Clin Psychol Sci Pract. 2003;10:144-156.

8. Kabat-Zinn J. Wherever You Go, There You Are: Mindfulness Meditation in Everyday Life. New York: Hyperion; 1994.

9. Teasdale JD, Segal ZV, Williams JM, Ridgeway VA, Soulsby JM, Lau MA. Prevention of relapse/recurrence in major depression by mindfulnessbased cognitive therapy. J Consult Clin Psychol. 2000;68:615-623.

10. Kuyken W, Byford S, Taylor RS, et al. Mindfulness-based cognitive therapy to prevent relapse in recurrent depression. J Consult Clin Psychol. 2008;76:966-978.

11. Segal ZV, Bieling P, Young T, et al. Antidepressant monotherapy vs sequential pharmacotherapy and mindfulness-based cognitive therapy, or placebo, for relapse prophylaxis in recurrent depression. Arch Gen Psychiatry. 2010;67:1256-1264.

12. Coelho HF, Canter PH, Ernst E. Mindfulness-based cognitive therapy: evaluating current evidence and informing future research. J Consult Clin Psychol. 2007;75:1000-1005.

13. Kingston T, Dooley B, Bates A, Lawlor E, Malone K. Mindfulnessbased cognitive therapy for residual depressive symptoms. Psychol Psychother. 2007;80:193-203.

14. Ma SH, Teasdale JD. Mindfulness-based cognitive therapy for depression: replication and exploration of differential relapse prevention effects. J Consult Clin Psychol. 2004;72:31-40.

15. Teasdale JD, Moore RG, Hayhurst H, Pope M, Williams S, Segal ZV. Metacognitive awareness and prevention of relapse in depression: empirical evidence. J Consult Clin Psychol. 2002;70:275-287.

16. Williams JM, Teasdale JD, Segal ZV, Soulsby J. Mindfulness-based cognitive therapy reduces overgeneral autobiographical memory in formerly depressed patients. J Abnorm Psychol. 2000;109:150-155.

17. Clarke K, Mayo-Wilson E, Kenny J, Pilling S. Can non-pharmacological interventions prevent relapse in adults who have recovered from depression? A systematic review and meta-analysis of randomised controlled trials. Clin Psychol Rev. 2015;39:58-70.

18. Strauss C, Cavanagh K, Oliver A, Pettman D. Mindfulness-based interventions for people diagnosed with a current episode of an anxiety or depressive disorder: a meta-analysis of randomised controlled trials. PloS One. 2014;9:e96110.

19. Meadows GN, Shawyer F, Enticott JC, et al. Mindfulness-based cognitive therapy for recurrent depression: a translational research study with 2-year follow-up. Aust N Z J Psychiatry. 2014;48:743-755.

20. Shallcross AJ, Gross JJ, Visvanathan PD, et al. Relapse prevention in major depressive disorder: mindfulness-based cognitive therapy versus an active control condition. J Consult Clin Psychol. 2015;83:964-975.

21. Williams JM, Crane C, Barnhofer T, et al. Mindfulness-based cognitive therapy for preventing relapse in recurrent depression: a randomized dismantling trial. J Consult Clin Psychol. 2014;82:275-286.

22. Manicavasgar V, Parker G, Perich T. Mindfulness-based cognitive therapy vs cognitive behaviour therapy as a treatment for non-melancholic depression. J Affect Disord. 2011;130:138-144.

23. Chiesa A, Castagner V, Andrisano C, et al. Mindfulness-based cognitive therapy vs. psycho-education for patients with major depression who did not achieve remission following antidepressant treatment. Psychiatry Res. 2015;226:474-483.

24. Eisendrath SJ, Gillung E, Delucchi KL, et al. A randomized controlled trial of mindfulness-based cognitive therapy for treatment-resistant depression. Psychother Psychosom. 2016;85:99-110. 
25. Lahera G, Bayón C, Bravo-Ortiz MF, et al. Mindfulness-based cognitive therapy versus psychoeducational intervention in bipolar outpatients with sub-threshold depressive symptoms: a randomized controlled trial. BMC Psychiatry. 2014;14:215.

26. Kazdin AE. Mediators and mechanisms of change in psychotherapy research. Annu Rev Clin Psychol. 2007;3:1-27.

27. Kazdin AE. Evidence-based treatment research: advances, limitations, and next steps. Am Psychol. 2011;66:685-698.

28. van der Velden AM, Kuyken W, Wattar U, et al. A systematic review of mechanisms of change in mindfulness-based cognitive therapy in the treatment of recurrent major depressive disorder. Clin Psychol Rev. 2015;37:26-39

29. Segal ZV, Williams JM, Teasdale JD. Mindfulness-Based Cognitive Therapy for Depression: A New Approach to Relapse Prevention. New York: Guilford Press; 2002.

30. Gu J, Strauss C, Bond R, Cavanagh K. How do mindfulness-based cognitive therapy and mindfulness-based stress reduction improve mental health and wellbeing? A systematic review and meta-analysis of mediation studies. Clin Psychol Rev. 2015;37:1-12.

31. van der Velden AM, Roepstorff A. Neural mechanisms of mindfulness meditation: bridging clinical and neuroscience investigations. Nat Rev Neurosci. 2015;16:439.

32. Kuyken W, Watkins E, Holden E, et al. How does mindfulness-based cognitive therapy work? Behav Res Ther. 2010;48:1105-1112.

33. Williams JM, Kuyken W. Mindfulness-based cognitive therapy: a promising new approach to preventing depressive relapse. Br J Psychiatry. 2012;200:359-360.

34. Crane C, Crane RS, Eames C, et al. The effects of amount of home meditation practice in mindfulness based cognitive therapy on hazard of relapse to depression in the Staying Well after Depression trial. Behav Res Ther. 2014;63:17-24.

35. Gibbons C, Felteau M, Cullen N, et al. Training clinicians to deliver a mindfulness intervention. Mindfulness. 2014;5:232-237.

36. Mental Health Foundation (2010) Be Mindful. Available from https:// www.mentalhealth.org.uk/sites/default/files/Mindfulness_report_2010. pdf. Accessed August 17, 2015.
37. Marx R, Strauss C, Williamson C. Mindfulness apprenticeship: a new model of NHS-based MBCT teacher training. Mindfulness. 2015;6:253-263.

38. Kuyken W, Hayes R, Barrett B, et al. Effectiveness and cost-effectiveness of mindfulness-based cognitive therapy compared with maintenance antidepressant treatment in the prevention of depressive relapse or recurrence (PREVENT): a randomised controlled trial. Lancet. 2015;386 (9988):63-73.

39. Kurdyak P, Newman A, Segal Z. Impact of mindfulness-based cognitive therapy on health care utilization: a population-based controlled comparison. J Psychosom Res. 2014;77:85-89.

40. Felder J, Dimidjian S, Beck A, Boggs JM, Segal Z. Mindful mood balance: a case report of web-based treatment of residual depressive symptoms. Perm J. 2014;18:58-62.

41. Beck A, Dimidjian S, Boggs J, Felder J, Segal Z. Internet delivered mindfulness-based cognitive therapy for reducing residual depressive symptoms: an open trial and quasi-experimental comparison to propensity matched controls. Clin Med Res. 2014;12:104.

42. Dimidjian S, Beck A, Felder JN, Boggs JM, Gallop R, Segal ZV. Web-based mindfulness-based cognitive therapy for reducing residual depressive symptoms: an open trial and quasi-experimental comparison to propensity score matched controls. Behav Res Ther. 2014;63:83-89.

43. Teasdale JD, Segal ZV. The Mindful Way Through Depression: Freeing Yourself from Chronic Unhappiness. New York: Guilford Press; 2007.

44. Williams JM, Penman D. Mindfulness: A Practical Guide to Finding Peace in a Frantic World. Paris: Hachette; 2011.

45. Taylor BL, Strauss C, Cavanagh K, Jones F. The effectiveness of self-help mindfulness-based cognitive therapy in a student sample: a randomised controlled trial. Behav Res Ther. 2014;63:63-69.

46. Shahar B, Britton WB, Sbarra DA, Figueredo AJ, Bootzin RR. Mechanisms of change in mindfulness-based cognitive therapy for depression: preliminary evidence from a randomized controlled trial. Int $J$ Cogn Ther. 2010;3:402-418.

47. Britton WB, Shahar B, Szepsenwol O, Jacobs WJ. Mindfulness-based cognitive therapy improves emotional reactivity to social stress: results from a randomized controlled trial. Behav Ther. 2012;43:365-380.
Psychology Research and Behavior Management

\section{Publish your work in this journal}

Psychology Research and Behavior Management is an international, peerreviewed, open access journal focusing on the science of psychology and its application in behavior management to develop improved outcomes in the clinical, educational, sports and business arenas. Specific topics covered in the journal include: Neuroscience, memory and decision making; Behavior

\section{Dovepress}

modification and management; Clinical applications; Business and sports performance management; Social and developmental studies; Animal studies. The manuscript management system is completely online and includes a very quick and fair peer-review system, which is all easy to use. Visit http://www. dovepress.com/testimonials.php to read real quotes from published authors. 